\title{
Fast-track drug approval in inflammatory bowel diseases
}

\author{
Konstantinos H. Katsanos a, Efstratios Koutroumpakis ${ }^{\mathrm{b}}$, Eftychia Giagkou ${ }^{\mathrm{a}}$, Zikos Malakos ${ }^{\mathrm{a}}$, \\ Eleni Almpania, Alexandros Skamnelos ${ }^{a}$, Dimitrios K. Christodoulou ${ }^{a}$
}

University of Ioannina School of Medical Sciences, Ioannina, Greece; Albany Medical College, Albany, New York, USA

\section{Abstract}

\begin{abstract}
Fast-track drug designation of safe regimens represents an emerging method of development and approval of new medications targeting debilitating diseases including inflammatory bowel diseases (IBD). The goal of accelerated drug approval pathways is to shorten the time between application and approval of therapies that treat diseases with significant morbidity and mortality. Recently, fasttrack drug approval approaches were supported by data deriving from central reading of images, a method of clinical data interpretation that has significantly benefited patients with gastrointestinal disorders. Biological agents and other emerging therapies in IBD represent "game-changing" or "treat-to-target" drugs and have satisfied quite successfully some of the patients' unmet needs. The development of biosimilars is an area where the Federal Drug Administration and the European Agency for Evaluation of Medicinal Products seem to have different approval processes. Biosimilars, including those for IBD, promise cost reductions and wide access to biologic therapies by patients, advantages similar to those already offered by generic drugs. Given the rapid development of IBD drugs and patients' needs, a consensus among the academic community, clinicians, researchers, sponsors, patients and regulatory authorities is required to standardize better the IBD trials and create a productive environment for fast-track approval of any "changing-game" IBD drug.
\end{abstract}

Keywords Crohn's disease, ulcerative colitis, inflammatory bowel diseases, fast track, drug approval, accelerated drug approval

Ann Gastroenterol 2016; 29 (4): 1-6

\section{Introduction}

Choosing the gold standard treatment of inflammatorybowel diseases (IBD) still represents a major challenge. Researchers and clinicians both now realize how IBD significantly increases patients' morbidity and negatively affects their overall quality of life. Therefore, there is a need for standardized approaches that will lead to the rapid development of new effective and safe treatments.

${ }^{a}$ Division of Gastroenterology, Department of Internal Medicine, Medical School, University of Ioannina School of Medical Sciences, Ioannina, Greece (Konstantinos H. Katsanos, Eftychia Giagkou, Zikos Malakos, Eleni Almpani, Alexandros Skamnelos, Dimitrios K. Christodoulou); ${ }^{b}$ Department of Internal Medicine, Albany Medical College, Albany, New York, USA (Efstratios Koutroumpakis)

\section{Conflict of Interest: None}

Correspondence to: Dimitrios K. Christodoulou, MD, PhD, Ass. Professor of Gastroenterology, Division of Gastroenterology, Department of Internal Medicine, Medical School, University of Ioannina School of Medical Sciences, 45110 Ioannina, Greece, Tel.: +30 26510 99639, Fax: +30 26510 07016, e-mail: dchristodoulou@gmail.com

Received 29 January 2016; accepted 26 April 2016; published online 3 June 2016

DOI: http://dx.doi.org/10.20524/aog.2016.0051
Over the last years, new approaches of mentoring drug research and testing have been developed. Among these methods the fast-track drug designation and subsequent approval of safe regimens represent an emerging drug development approach in IBD treatment [1]. In cancer therapies, recent fast-track drug approvals were strongly supported by data derived from central reading of images, a method of clinical data interpretation that has significantly contributed in gaining substantial benefits for the patients [2].

Biological and other emerging IBD therapies currently represent a treat-to-target approach, a concept that cancer therapies have already adopted for many years. In this era, the challenge of treating IBD is to early access new drugs that ensure bowel healing without limiting patient safety [3]. The present review focuses on the concepts of fast-track and accelerated drug approval and how these approaches benefit IBD patients.

\section{Search strategy}

A comprehensive literature search using the terms and variants of inflammatory bowel diseases (IBD, ulcerative colitis, Crohn's disease) was performed in December 2014 within Pubmed, Embase and Scopus and restricted to human studies and EMBASE. The search included the terms 
inflammatory bowel diseases OR ulcerative colitis OR Crohn's disease AND fast-track OR accelerated drug approval. Studies were included if they were published in any language and if related to fast-track or accelerated drug approval in IBD as well as other diseases. Additionally, references from relevant papers were hand searched for other appropriate studies and from the FDA and the EMEA official websites. Fast-track drug approval in IBD

\section{The rationale for fast-track programs in IBD}

The goal of accelerated pathways is to shorten the time between application and approval of therapies that treat diseases with significant morbidity and mortality. There is always a concern that regulations sometimes hamper drug development and delay approval processes even for drugs for which there are only a few alternatives that are effective. The processes are further delayed by the excessive and duplicative costs that pharmaceutical companies have to cover in their efforts to meet clinical research requirements and high standards of safety and effectiveness. Until now, accelerated approval programs supported by both the United States Food and Drug Administration authorities (FDA) and the European Medicines Agency (EMA) have been mainly applied by pharmaceutical companies to the development of Oncology drugs [4-6].

Accelerated approval programs have incredibly benefited cancer patients when introduced and implemented properly. On the contrary, thousands of IBD patients across USA and Europe, who need new treatment options, are still facing delays of many months or years before they access those new treatments, which can be vital for them, given that IBD may rapidly progress to severe or even life-threatening forms [7-8].

\section{Applying fast-track programs in IBD}

In 1997, the Food and Drug Administration Modernization Act directed FDA to create a mechanism whereby important new drugs could be approved faster than following the standard and priority review programs already in place, including the "accelerated approval" and the "priority review" programs. The Accelerated Approval Program was instituted by FDA to allow for earlier approval of drugs. It is appropriate for medications that demonstrate an effect on a surrogate, or intermediate endpoint, which is reasonably likely to predict clinical benefit. A valuable endpoint could be a marker, such as a laboratory test, a computed tomography or magnetic resonance finding, an objective sign at physical examination or any other test that is clinically meaningful. It is expected that in several instances an objective endpoint can significantly shorten the time needed prior to receiving FDA approval.

A priority review is a review conducted with a Prescription Drug User Fee Act (PDUFA) goal date of 6 months. In 1992 a law in USA allowed the FDA to collect fees from pharmaceutical industries to finance the new drug approval logistics.
According to that action, the FDA was entitled to collect fees from pharmaceutical industries at the time that a new drug application or biologics license application was submitted. To further promote and further support the collection of such fees, the FDA was required to speed certain activities. For example, the priority review shortens FDA review process for a new drug from ten to six months. This priority review process was applicable for drugs showing reliable characteristics in both safety and effectiveness compared to an already existing therapy. Rolling review means that a drug industry can submit its new drug application for review by FDA in parts, rather than waiting for the entire application to be completed and then subsequently submitted for review.

The 'fast-track' designation was then added to the other already approved FDA programs. Fast track is a process designed to facilitate the development, and expedite the review of drugs treating severe conditions and that successfully address unmet medical needs. The purpose is to get important new drugs to the patient earlier. Fast track applies to a broad range of serious conditions. Fast-track designation must be requested by the drug company, which also has to submit all the supporting documentation for the product and its proposed indications for use. This request can be initiated at any time during the drug development process. In general, the fast-track designation will be considered appropriate during Phase 2 or 3 clinical trials of the drug. The request will be reviewed by the FDA and within 60 days a report will be available stating whether the conditions for fast-track designation have been met or not [9].

By definition, FDA fast-track designation facilitates the development of drugs intended to treat serious or lifethreatening conditions. Determining whether a disease is serious or not can be subjective, but generally the concept is based on whether the drug will have an impact on factors including survival, or the probability that the disease, if left untreated, will progress to a life-threatening status or even a fatal outcome. Addressing an unmet medical need by a medication means that the medication intents to treat or prevent a disease that does not have a current treatment. If there are existing therapies, a fast-track eligible drug must show clear advantage over available treatment, such as superior effectiveness and/or better safety [4-8].

When a drug receives the fast-track designation label a frequent communication between FDA and the drug company is expected to happen. Several other procedures are encouraged throughout the entire drug development and review process, including more frequent written correspondence and more frequent meetings of the sponsor with FDA. The frequent communication aims to outline the drug's development plan, ensure the collection of appropriate data needed to support faster drug approval, and resolve any issues that can potentially delay early access to the drug by patients $[10,11]$.

Since 2001, the European Commission has started a fasttrack approval program for the European Agency for Evaluation of Medicinal Products (EMEA). The whole process is focusing on drugs of therapeutic innovation or of major public health interest. The Committee for Proprietary Medicinal Products (CPMP), established under the EMEA, is responsible for such 
an accelerated review process. The requirements and time frames for approval of a drug under the accelerated review process are similar to those utilized by the FDA. Eligibility for fast track or any other type of accelerated approval is based on endpoints that predict substantial clinical improved outcomes. For instance, a drug that promises to extend the survival of patients with cancer can be approved even if it has only been shown to shrink tumors in a clinical trial. Shrinking tumors is a surrogate endpoint because it is a meaningful outcome and an indirect measurement of the drug's effectiveness [6,11]. The evidence based equivalent for IBD is difficult to define. The gold standard would probably be complete histological healing. Complete mucosal healing with absence of any signs of other intestinal or/and abdominal damage would be a meaningful outcome in clinical practice.

Of note, final approval of a drug based on such kind of endpoints is given if the confirmatory phase IV trial that

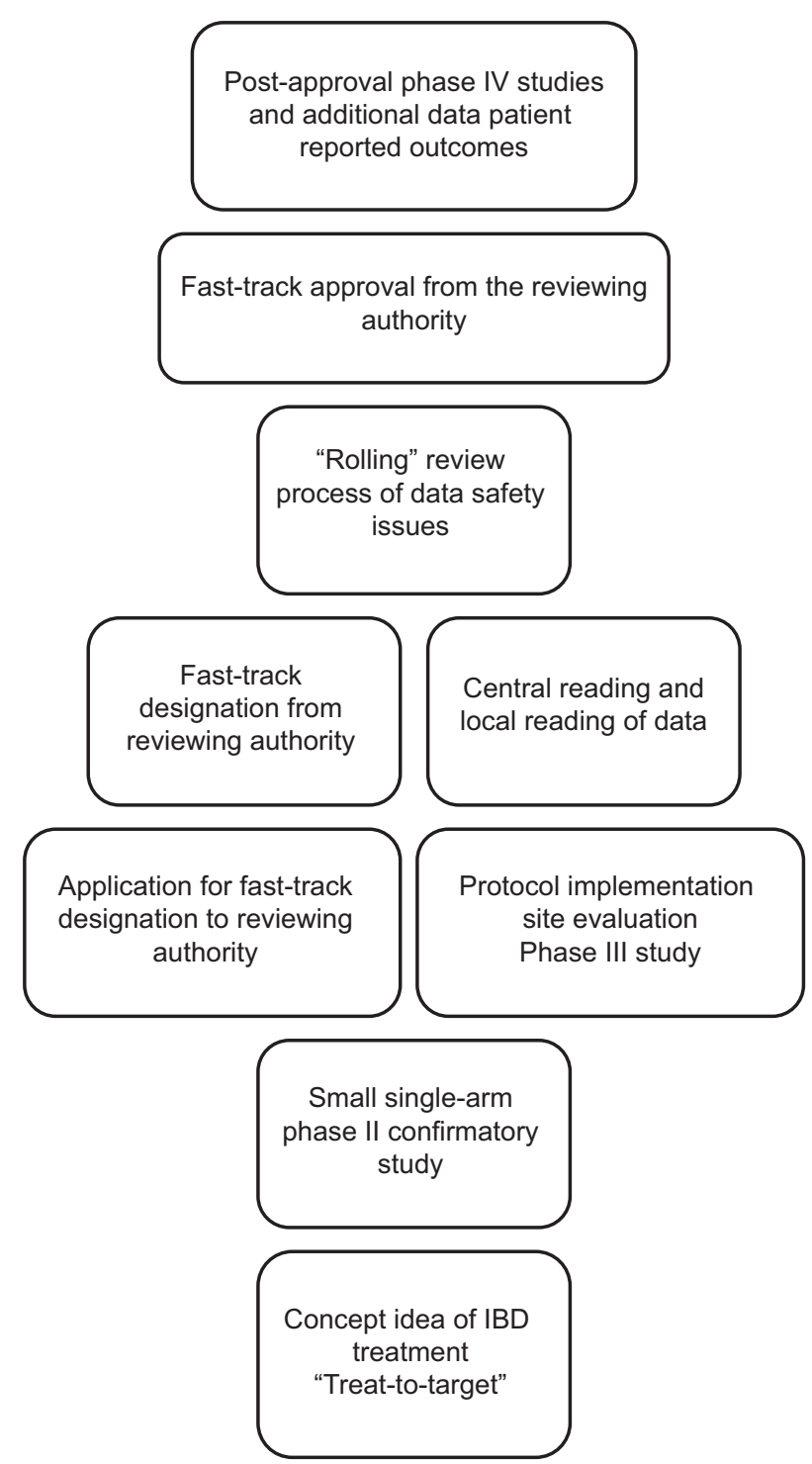

Figure 1 The road of fast-track drug approval in inflammatory bowel diseases (IBD) using central reading of data follows initial approval provides data verifying the clinical benefit. If the confirmatory trial fails to show clinical benefit, the FDA can remove the drug from the market (Fig. 1).

Over the past decade, have been many attempts to harmonize all such fast-track processes of the FDA and the EMEA. Very soon, it became evident that there are many similarities but also multiple differences between these two agencies in several key points. These points include the time from application to drug approval, the phrasing of drug indications as well as the ability of patient access to therapies in various countries. Over these last years, mutual understanding of the regulatory mechanisms in the US and the European Union (EU) is expected to facilitate drug approval process to benefit patients who urgently need those therapies. For example, the approval processes of chemotherapeutic agents have been facilitated by the coordination of regulatory mechanisms between the EMA and the FDA [12-14].

An interesting question would be whether the currently -or soon to be- approved IBD drugs would have met the criteria for fast-track review and what would the outcomes have been. Although it is difficult to predict, the authors' opinion is that fast track is a promising but probably premature concept for most aspects of IBD treatment at present time. Large studies using well-defined treatment targets such as mucosal or histological healing as their primary endpoints are required. Furthermore, IBD drugs have to be carefully re-evaluated in clinical practice and the "yellow cards" reporting any possible or probable drug-related adverse reaction should be meticulously completed by every physician treating patients with IBD.

\section{Orphan product framework and IBD}

Based on the US Orphan Drug Program, drugs, biologics, medical devices and medical foods can be characterized as "orphans". A product concerning a rare disease or infrequent condition can be given the orphan status provided that the prevalence threshold of the so-called "low incidence" is set at 7.5 per 10,000 people. For example, in the USA a disease is rare when it affects less than 200,000 individuals. However, a drug may still be characterized as "orphan" if some certain financial criteria are fulfilled [15]. Within the EMEA, the Committee for Orphan Medicinal Products (COMP), is responsible for reviewing applications for orphan drugs. It is important to stress herein that most of the applications are reviewed under exceptional circumstances as the reviewing committee accepts a priori that that the majority of the applicants may not be able to provide solid data concerning efficacy and safety of the drug under normal conditions of use $[6,10]$.

Products treating IBD patients in general are not appropriate for orphan drug status based on the above definition. However, there are distinct groups of IBD patients such as those with pouchitis, severe malabsorption due to short bowel, and other severely disabling complications in whom disease significantly affects life expectancy and quality of life and for whom orphan drug status may apply. 


\section{Concerning issues about fast track}

Several issues have been raised about the fast-track and the accelerated approval process. These issues include the firm validation and stability of the endpoints used, and the assessment of expected clear benefit, as well as the need for further confirmatory studies. The majority of accelerated approvals granted to date have been based on phase II trials, which represent a level of evidence less reliable, compared to trials required for full approval.

Selection bias always represents an important concern. Therefore, promising but potentially misleading results from phase II trials require subsequent larger phase III trials for solid confirmation of the results on efficacy and safety. In general, phase II trials are considered to be sufficient for fast-track FDA approval of treatments for severe diseases, as soon as the drug producer has also applied for extensive post-marketing "phase IV" confirmatory trials $[1,2]$.

It is also true that misleading negative results for a beneficial drug from phase II trials would not be followed up in subsequent studies. Safety issues are of major importance. The risk of approving a "toxic placebo" increases as the standards of approval are lowered [9]. These considerations strongly suggest that the results of phase II trials in new fasttrack approved drugs need to be interpreted with caution and that their introduction in the fast-track process can be often very challenging. To reassure quality of such approval process, randomized trials comparing a new drug against a control drug or a placebo therapy seem to guarantee the best protection against the possible risks of misleading results that may be inherent in phase II trials with restricted number of observations.

\section{Cost of care in fast track}

The cost of IBD care is increasing worldwide as IBD incidence and prevalence are rapidly increasing in most of the counties. The increasing cost of IBD treatment is resulting from the significant costs of new therapeutic molecules to the market as well as from the significant morbidity that IBD is causing. To reduce the costs of drug development in IBD and to facilitate fast-track approval of effective IBD therapies, efforts need to be made to optimize the regulatory process. This is very important for the fast-track introduction of new drugs, which is mainly based on improving our knowledge and advancing our understanding of the intestinal epithelial immunology and the molecular targets of bowel inflammation [16].

\section{Biosimilars and fast-track approval in IBD}

The process of development of biosimilars clearly demonstrated that FDA and the EMEA have several differences. Biosimilars, including those for IBD, promise cost reductions and wide access to more expensive therapies by the patients in countries with lower mean incomes. However, because the manufacturing of biologic agents is very different from that of small-molecule agents, it is questionable if the standards already existing in generic drugs could also be applicable for biosimilars [17].

The EU developed a regulatory strategy for biosimilars in 2004 and in 2009 the development of a biosimilar regulatory act was also implemented in the US. Since then, multiple biosimilars have become available based on an accelerated approval approach similar to their predecessor biologic drug. These regulatory strategies do consider biosimilars as agents that practically demonstrate no differences in terms of efficacy and safety compared with the original predecessor drug. However, it still remains challenging whether a comparable standard to assess biosimilar effectiveness as the reference product really exists [18].

The long-term impact of biosimilars on the cost of IBD care is yet to be determined and is dependent on the overall manufacturing costs of biosimilars. On the other hand, it is anticipated that industries producing biosimilar products will probably lower their prices to remain competitive. Key will be the timing of the regulatory processes, since the more costly the process is, the smaller the price difference and the less likely for the product to be introduced in the IBD market will be. Safety and efficacy remain extremely important for any new agent involved in this frame of biosimilar approving $[19,20]$.

Drug-related official authorities, such as EMA and FDA, apply their own rules for the process of demonstration of the similar nature of two biological products in terms of safety and efficacy. According to this regulatory process of each of these authorities, detailed studies showing that the biosimilar product is highly similar to the original molecule are extremely important. An example is Remsima (or inflectra), for which extensive physicochemical and biological characterization of the product itself and its reference product Remicade (infliximab) was conducted to demonstrate their highly similar properties in terms of efficacy and safety.

During the first steps of biosimilar approval, the initial complexity of production of their original biological molecules has led to requests for extensive reports on efficacy and safety data to approve the biosimilar product of an original biologic drug. Through the years, this has been progressively replaced by sensitive assays to detect significant differences in dose. It is important to state herein that the safe application of biologics and their biosimilars largely depends on their appropriate use by gastroenterologists and other healthcare physicians. Finally, the introduction of biosimilars in the drug market requires careful implication of long-term pharmacovigilance, as this has been also been the case in the original biological molecules after their introduction in the IBD drug market.

\section{Fast track for "treat-to-target" and "game-changing" drugs}

Targeted therapies are those tailored to a patient's genetic makeup. Several drug companies have been focusing on the production of so-called immunotherapy medications, which intend to modify the "brakes" on the immune system. For example, identifying the role of BRAF gene in patients with melanoma led to the development of the unprecedented 
"treatment concept" of BRAF inhibitors. BRAF is a human gene that makes a protein called B-Raf. Interestingly, FDA fasttracked the approval of vemurafenib for use in people with the $B R A F$ V600E polymorphism in less than four months after it was submitted. This was a huge boost to the research efforts of such drug makers [21].

Since 2011, FDA has approved seven treatments for advanced melanoma that promote an immune response including antibodies designed to target the programmed death-1 (PD-1) pathway. The anti-PD-1 drugs that have been produced so far have been developed at a high speed, needing only three years from the first clinical trials until approval. Among these anti-PD-1 drugs, lambrolizumab received a breakthrough therapy designation from FDA following promising results from only a small single-arm study. It is noteworthy that in the confirmatory lambrolizumab study the investigator-reported data was compared with a blind central review to confirm drug effectiveness in a solid way. In that particular study, the efficacy analysis included two endpoints: first, the overall responses derived from investigator-reported data, and second, all overall responses also derived from independent, centralized and blinded radiologic image review by experts. Of importance, there was a high agreement on response rates across all doses, between the investigators and the central reviewers [22].

However, it may happen that a "game-changing" or "treat-to-target" drug succeeds a fast-track designation by demonstrating a low but clear response rate, and in subsequent larger trials fails to show benefit. Therefore, the medications that have received fast-track designation can initially only be approved for use as an alternative in patients who show no response to prior therapies or as rescue or salvage therapy under an expanded access program. It is important to stress here that the concept of treat-to-target as a means to enhance patient outcome is a therapeutic strategy and is not directly linked to approval of specific biologic drugs per se.

\section{New concepts in fast-track drug approval in IBD}

\section{Fast track in IBD supported by endoscopy images}

Where appropriate, FDA and EMEA may grant designation to the development of a new use of an already approved drug that is effectively and safely addressing a serious condition and demonstrates the potential to address unmet medical needs. IBD affects large populations including young adults in their productive age and also children. Furthermore, it is associated with unacceptable morbidity and mortality. With that being said, IBD patients have still several important unmet clinical needs that urgently require effective treatment. More specifically, there is a real-life need of drugs for IBD patients who do not respond to previous IBD therapies, or experience loss of response, or have intractable disease accompanied by severe morbidity and mortality. As physicians started appreciating those unmet needs, the endpoints of IBD studies changed from more general to more objective outcomes [23].
Interestingly, the inconclusive results of studies that did not utilize imaging in the assessment of their outcomes consisted important triggers in the search of new "treat-to-target" therapies in IBD. Studies relying only on patients' quality of life or patient-reported activity indexes and outcomes are not practical to clinicians and often include very subjective assessments. In contrast, IBD studies that use objective measures such as images or videos to record outcomes create a stronger unbiased basis for assessing the effectiveness of drugs such as infliximab and adalimumab in inducing bowel mucosa healing [24]. We should mention here that all such studies carry an inherent difficulty of recruiting and scoping patients with IBD as opposed to studies utilizing more convenient imaging modalities such as X-rays, computed tomography scans or magnetic resonance imaging.

\section{Basic concepts of modern drug development in IBD}

Modern drug development in IBD should rely upon endoscopic findings, patient-reported symptoms, radiologic assessment of the disease burden (MRI or U/S for bowel wall thickness, abscesses and fistulas) and histology. Historically, assessment of mucosal healing has been demonstrated by some routine images of the bowel. In recent years, there has been a strong tendency of referring those images or even endoscopy videos for central reading. These emerging new concepts have already been adopted by several studies evaluating new therapeutic molecules and new endoscopy scoring systems in IBD. This centralization is based on the assessment of full-length videos of colonoscopies or/and sigmoidoscopies, in order to most closely and objectively evaluate the clinical site [25]. This centralization of readings has also been applied to many other medical specialties. In fact, central reading has been used so far in 35 studies in Ophthalmology, 25 studies in Cardiovascular and Pulmonary diseases, 14 studies in Rheumatology and Orthopedics, 7 studies in Neurology and several other studies in various medical specialties [supplementary Table and supplementary references 1-105].

According to this data, there is clear evidence that central reading of images increases the quality of clinical trial data in IBD and other fields. However, the link by which central reading may enhance fast-track approval apart from its impact on robust clinical trial output is not always clear. In fact, FDA, EMEA and other drug-approving authorities have not implemented central reading of images as a mandatory prerequisite for fast-track drug approval.

\section{Patient-reported outcomes (PROs)}

Maximization of long-term health-related quality of life in patients with IBD can be achieved only through control of symptoms. Both EMA and FDA recognize the value of PROs as mandatory items when determining the efficacy of medical therapies and considering them for ultimate approval. When evaluating PROs, EMA appreciates multiple domains for a 
general assessment of health related quality of life, while FDA focuses more on symptom-specific measures. Authorities approving new drugs often do consider PROs as an additional secondary endpoint of clinical efficacy rather than part of a primary assessment which is crucial for drug approval. Both agencies recognize the value of these reported outcomes in showing the patient's perspective to the new therapeutic molecules. Indeed, endoscopy and histology do not always correlate with symptoms $[6,7,14]$.

\section{The future in IBD drug development}

The gold standard approach of imaging reading and the gold endpoint in IBD trials are yet to be determined. Over the years, it has been shown that testing of 'treat-to-target' drugs that seek fast-track designation can be achieved by small onearm studies versus large-scale studies with multiple endpoints. Recently, fast-track approval of many "changing-game" drugs was based on phase II rather than phase IV trials. Important components of fast-track designation and approval of a new drug in IBD include not only disease severity and disease extension but also patient-reported symptoms and their unmet needs. It is important that patient organizations communicate their unmet needs and the real burden of their disease.

Given the rapidly evolving technology leading to the development of new breakthrough medication targets and the patients' unmet needs, a consensus among academic community, clinicians, researchers, sponsors, patients and regulatory authorities is required on the best approach of standardizing IBD trials and creating a productive environment for fast-track approval of any "changing-game" IBD drug.

\section{References}

1. Hoag H. Drug development: a chance of survival. Nature 2014;20;515:S118-S120.

2. Trotta F, Leufkens HG, Schellens JH, et al. Evaluation of oncology drugs at the European Medicines Agency and US Food and Drug Administration: when differences have an impact on clinical practice. J Clin Oncol 2011;29:2266-2272.

3. Bouguen G, Levesque BG, Feagan BG, et al. Treat to target: a proposed new paradigm for the management of Crohn's disease. Clin Gastroenterol Hepatol 2015;13:1042-1050.

4. Fast track, breakthrough therapy, accelerated approval and priority review. From: http://www.fda.gov/forpatients/approvals/fast/ ucm20041766.htm

5. Illman J. News: Stat bite: cancer drug approvals in Europe and the United States, 1995-2001. J Natl Cancer Inst 2001;93:1682-1683.

6. http://www.ema.europa.eu/docs/en_GB/document_library/ Scientific_guideline/2009/09/WC500003517.pdf

7. FDA Guidance for Industry: Expedited programs for serious conditions-drugs and biologics - 5/2014; Appendix 2; Appendix 3 - (CDER MAPP 6020.3); CBER SOPP 8405; Appendix 4.

8. FDA, fast track, breakthrough therapy, accelerated approval and priority review: Expediting availability of new drugs for patients with serious conditions. http://www.fda.gov/ForPatients/ Approvals/Fast/ucm405447.htm

9. Lamont EB, Hayreh D, Pickett KE, et al. Is patient travel distance associated with survival on phase II clinical trials in oncology? J Natl Cancer Inst 2003;95:1370-1375.

10. http://www.ema.europa.eu/docs/en_GB/document_library/ Scientific_guideline/2009/09/WC500003637.pdf.

11. Bottomley A, Jones D, Claasens L. Patient-reported outcomes: assessment and current perspectives of the guidelines of the food and drug administration and the reflection paper of the European Medicines Agency. Eur J Cancer 2009;45:347-353.

12. FDA launches 7 initiatives with European drug regulators. Available from: http://www.cancernetwork.com/articles/fdalaunches-7-initiatives-european-drug-regulators.

13. Roberts SA, Allen JD, Sigal EV. Despite criticism of the FDA review process, new cancer drugs reach patients sooner in the United States than in Europe. Health Aff 2011;7:1375-1381.

14. Downing NS, Aminawung JA, Shah ND. Regulatory review of novel therapeutics-comparison of three regulatory agencies. N Engl J Med 2012;366:2284-2293.

15. US orphan drug program www.fda.gov/orphan/index.htm

16. Howie LJ, Hirsch BR, Abernethy AP. A comparison of FDA and EMA drug approval: implications for drug development and cost of care. Oncology 2013;27:1195.

17. Development and approval processes: biologics. Available from: http://www.fda.gov/BiologicsBloodVaccines/Development ApprovalProcess/BiologicalApprovalsbyYear

18. Megerlin F, Lopert R, Taymor K, Trouvin JH. Biosimilars and the European experience: implications for the United States. Health Aff 2013;32:1803-1810.

19. Hirsch BR, Lyman GH. Will biosimilars gain momentum? J Natl Compr Canc Netw 2013;11:1291-1297.

20. Guidance for industry. Biosimilars: questions and answers regarding the implementation of the Biologics Price Competition and Innovation Act of 2009. Available from: http://www.fda.gov/ downloads/Drugs/GuidanceComplianceRegulatory Information/ Guidances/UCM273001.pdf

21. Beazley-Long N, Gaston K, Harper SJ, Orlando A, Bates DO. Novel mechanisms of resistance to vemurafenib in melanoma - V600E B-Raf reversion and switching VEGF-A splice isoform expression. Am J Cancer Res 2014;5:433-441.

22. Hamid O, Robert C, Daud A, et al. Safety and tumor responses with lambrolizumab (anti-PD-1) in melanoma. $N$ Engl $J$ Med 2013;369:134-144.

23. Cooney R, Warren B, Altman D, et al. Outcome measurement in clinical trials for ulcerative colitis: towards standardization. Trials 2007;8:17.

24. Casellas F, Barreiro de Acosta M, Iglesias M, et al. Mucosal healing restores normal health and quality of life in patients with inflammatory bowel disease. Eur $J$ Gastroenterol Hepatol 2012;24:762-769.

25. Krzeski P, O'Leary DH, Zabbatino S. Lessons learned from the use of central endoscopy review in inflammatory bowel disease trials. http://www.medpace.com/Offers/GI_CentralReader/Medpace_ GI_Imaging_IBD_White_paper_May2013.pdf 